\title{
Secret interactions of eV-scale sterile neutrinos and the diffuse supernova neutrino background flux
}

\author{
Y. S. Jeong \\ Theory Division, CERN, 1211 Geneve 23, Switzerland \\ E-mail: yuseon.jeong@cern.ch

\section{S. Palomares-Ruiz} \\ Instituto de Física Corpuscular (FIC), CSIC-Universitat de València, E-46071 València, Spain \\ E-mail: sergiopr@ific.uv.es
}

\section{H. Reno*†}

Department of Physics and Astronomy, University of Iowa, Iowa City, IA 52242, USA

E-mail: mary-hall-reno@uiowa.edu

\section{Sarcevic}

Department of Physics, University of Arizona, Tucson, AZ 85721, USA

E-mail: ina@physics.arizona.edu

For a sterile neutrino with an $\mathrm{eV}$-scale mass and vacuum mixing angle of order $\theta_{0} \simeq 0.1$, our evaluation of big bang nucleosynthesis and cosmic microwave background constraints allow a new gauge sector for sterile neutrino "secret interactions" with a gauge boson mass between a few $\mathrm{keV}$ and $\sim 100 \mathrm{keV}$ and sterile gauge boson couplings of order $g_{s} \simeq 10^{-4}-10^{-2}$. Relic sterile neutrinos with $m_{s}=1 \mathrm{eV}$ that interact via gauge bosons with a mass between $5-8 \mathrm{keV}$ and couplings in this range would distort the spectrum of the diffuse supernova neutrino background flux. We explore the potential for the distortion to be measured at DUNE and Hyper-Kamiokande.

The 20th International Workshop on Neutrinos (NuFact2018)

12-18 August 2018

Blacksburg, Virginia

*Speaker.

†This work was supported in part by the US Department of Energy (DE-SC-0010113, DE-SC-0010114, DE-SC0002145 and DE-SC0009913), the Ramón y Cajal program, the Spanish MINECO (FPA2017-84543-P, FPA201454459-P and SEV-2014-0398), the Generalitat Valenciana (PROMETEOII/2014/049), the EU Horizon 2020 (Marie Skłodowska-Curie No 690575 and No 674896) and the Portuguese FCT (PEst-OE/FIS/UI0777/2013). 


\section{Introduction}

The theory of three flavors of massive neutrinos is successful in its description of neutrino oscillation phenomena. Nevertheless, the LSND [1], MiniBoone [2] and reactor anomalies [3] can be accommodated with the introduction of an $\mathrm{eV}$-scale massive sterile neutrino with a vacuum mixing angle with active neutrinos of order $\theta_{0} \sim 0.1$. Searches for evidence of sterile neutrinos in experiments using accelerator beams and reactors are on-going [4]. Here we discuss potential signals in astrophysics that could reveal the existence of $\mathrm{eV}$-scale sterile neutrino relics from the Big Bang, in particular, where "secret interactions" mediate interactions with sterile neutrinos but not ordinary neutrinos, except through neutrino mixing [5,6]. The secret interactions, in some regions of parameter space, will be evident in absorption dips in the astrophysical neutrino flux.

The idea of probing the relic neutrino density with absorption dips is not new. Weiler proposed to measure the Standard Model (SM) relic neutrinos through resonant production of $Z^{0}$ bosons [7]. Neutrinos of extremely high energies, of order $10^{13} \mathrm{GeV}$ incident on fixed target neutrinos with masses $\sim 0.1 \mathrm{eV}$, are required to produce on-shell $Z^{0}$ bosons. For secret interactions with $\mathrm{keV}$ scale gauge boson $\phi$ mediators and $\mathrm{eV}$-scale sterile neutrinos, the relevant astrophysical neutrino energy for resonant $\phi$ production is $E_{V} \sim 10 \mathrm{MeV}$. One source of astrophysical neutrinos in this energy range are supernovae. In this proceedings, we describe an evaluation of the absorption dips in the diffuse supernova neutrino background (DSNB) flux from neutrino interactions with relic eVscale sterile neutrinos with sterile sector secret interactions mediated by $\mathrm{keV}$-scale $\phi$ bosons. We report on the potential for DUNE and Hyper-Kamiokande (HK) to see evidence of these absorption dips in the DSNB spectra [5].

\section{Results}

Cosmological constraints on the sterile sector with secret interactions come from big bang nucleosynthesis (BBN) and the cosmic microwave background (CMB) [5, 6]. In consideration of the sterile sector, self-interactions cannot be taken as effective four-fermi interactions. We have assumed a $V-A$ sterile neutrino- $\phi$ interaction term with coupling constant $g_{s}$. Sterile-active neutrino mixing provides the opportunity for sterile neutrinos to recouple with the SM sector and violate the $\mathrm{BBN}$ bound on the number of equivalent active neutrino species, $N_{v}<3.2$. The cosmic microwave background constraints come from neutrino free-streaming considerations. We require that sterile neutrinos be decoupled from active neutrinos at $T_{V} \sim 1 \mathrm{eV}$, although recoupling with active neutrinos after BBN but before the cosmic microwave background epoch is permitted. We find a band of allowed $\left(g_{s}, m_{\phi}\right)$ parameter space for $m_{s} \sim 1 \mathrm{eV}$ and $\theta_{0}=0.1$. To be testable with the DSNB flux, $m_{\phi}=5-8 \mathrm{keV}$, for which $g_{s} \simeq 10^{-4}-10^{-2}$ is permitted. We take as our standard choice $g_{s}=10^{-4}$. The cosmological constraints on these parameters continue to be a topic of interest [8]. We note that constraints on a sterile sector from CMB limits are difficult to obtain consistently since SM simulations are inputs.

Examples of our results for the impact of absorption dips are shown in fig. 1 for the normal hierarchy of neutrino masses for DUNE (40 kt LAr) and HK (187 kton) [5]. Here, we have used the supernova formation rate based on ref. [9] and supernova energy spectrum according to ref. [10]. Gaussian energy resolutions are assumed in the evaluation of the differential event rates. 

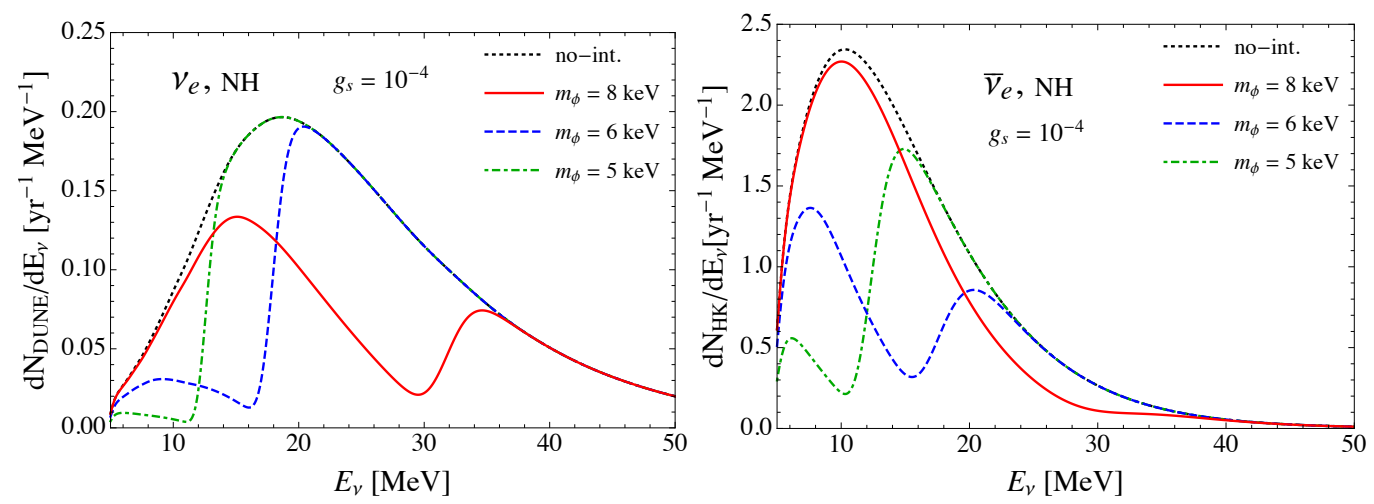

Figure 1: For the normal hierarchy, left: differential event rates expected at the 40 kton DUNE LAr detector from $v_{e}$ charged-current interactions off ${ }^{40} \mathrm{Ar}$, and right: differential event rates for one $187 \mathrm{kton}$ HyperKamiokande tank from $\bar{v}_{e}$ inverse beta decay off water [5] as a function of reconstructed neutrino energy based on a Gaussian energy resolution function of width $\sigma / E_{v}=0.05(0.10)$ for DUNE (HK).

The dashed black lines show the differential event rate without secret interactions, and the colored lines show the absorption effect of sterile neutrino interactions with $m_{\phi}=5,6$ and $8 \mathrm{keV}$. The figures illustrate the measurement potential for DUNE and HK. For the normalization assumed here, measurements will be challenging, however, we note that there are large uncertainties in the normalization of the DSNB flux.

\section{References}

[1] C. Athanassopoulos et al. [LSND Collaboration], Phys. Rev. Lett. 75, 2650 (1995) [nucl-ex/9504002]; Phys. Rev. Lett. 81, 1774 (1998) [nucl-ex/9709006].

[2] A. A. Aguilar-Arevalo et al. [MiniBooNE Collaboration], Phys. Rev. Lett. 98, 231801 (2007) [arXiv:0704.1500 [hep-ex]]; Phys. Rev. Lett. 105, 181801 (2010) [arXiv:1007.1150 [hep-ex]].

[3] G. Mention, M. Fechner, T. Lasserre, T. A. Mueller, D. Lhuillier, M. Cribier and A. Letourneau, Phys. Rev. D 83, 073006 (2011) [arXiv:1101.2755 [hep-ex]].

[4] S. Böser, C. Buck, C. Giunti, J. Lesgourgues, L. Ludhova, S. Mertens, A. Schukraft and M. Wurm, arXiv:1906.01739 [hep-ex].

[5] Y. S. Jeong, S. Palomares-Ruiz, M. H. Reno and I. Sarcevic, JCAP 1806, no. 06, 019 (2018) [arXiv:1803.04541 [hep-ph]].

[6] S. Hannestad, R. S. Hansen and T. Tram, Phys. Rev. Lett. 112, no. 3, 031802 (2014) [arXiv:1310.5926 [astro-ph.CO]]; B. Dasgupta and J. Kopp, Phys. Rev. Lett. 112, no. 3, 031803 (2014) [arXiv:1310.6337 [hep-ph]]; J. F. Cherry, A. Friedland and I. M. Shoemaker, arXiv:1411.1071 [hep-ph]; X. Chu, B. Dasgupta and J. Kopp, JCAP 1510, no. 10, 011 (2015) [arXiv:1505.02795 [hep-ph]].

[7] T. J. Weiler, Phys. Rev. Lett. 49, 234 (1982).

[8] X. Chu, B. Dasgupta, M. Dentler, J. Kopp and N. Saviano, arXiv:1806.10629 [hep-ph].

[9] H. Yuksel, M. D. Kistler, J. F. Beacom and A. M. Hopkins, Astrophys. J. 683, L5 (2008) [arXiv:0804.4008 [astro-ph]].

[10] M. T. Keil, G. G. Raffelt and H. T. Janka, Astrophys. J. 590, 971 (2003) [astro-ph/0208035]. 